researchers annealed the samples in $1 \mathrm{~atm}$ flowing oxygen at $900^{\circ} \mathrm{C}$. Following the implantation with one, two, and then all of the elements, secondary ion mass spectrometry depth profiles were taken of both the annealed and unannealed samples. The researchers found that when magnesium was implanted on its own, it did not diffuse after annealing. They also found that the magnesium moved most when it was co-implanted with just sodium, and less when aluminum was also implanted. The researchers said, "These findings corroborate the roles of sodium, magnesium, and aluminum in the oxidation behavior of NBD 200 silicon nitride ceramic."

PAMELA JOHNSON

\section{Protein Promotes Nucleation in Water Microdroplets}

Ice crystallization in micro-sized water droplets plays an important role in nature. Researchers at the National University of Singapore have made a quantitative measurement of the interfacial effect of impurities on ice nucleation kinetics. Using microdroplet suspension to minimize the effects of the container and foreign particles, Du Ning and X.Y. Liu found that the protein lysozyme promotes freezing as a result of its effects on the interfacial kinetics of nucleation. The crystallization of microdroplets has significant implications for natural processes or systems such as climatic systems and the freezing and antifreezing processes within plants and animals.

As described in the July 15 issue of Applied Physics Letters, a 0.4- $\mu 1$ droplet of deionized water was suspended between two layers of oil of different densities. To study the nucleation kinetics, the researchers measured the amount of time it took for an observable amount of the new phase to appear at several different temperatures. This induction time included both the time for crystals to nucleate and the time for them to grow to an observable size. However, because ice crystals grow much faster than they nucleate, the growth time can be neglected, and the time measured in the experiment was considered to be the nucleation time only.

At a constant nucleation rate, a smaller water droplet has a longer induction time. In bulk water, a small change in volume during the experiment has a negligible effect, since the total volume is so large. However, for microdroplets, the change in volume can double the induction time. The researchers included the size effects by multiplying the induction time by the volume of the water droplet, allowing a precise and reproducible measurement of the nucleation kinetics. The effects of pro- teins on ice nucleation kinetics was also studied.The researchers found that the addition of lysozyme at $0.05 \mathrm{wt} \%$ to the droplets had two effects. First, it raised the energy barrier for removing impurity molecules from the surface, which inhibited nucleation. Second, the lysozyme decreased the interfacial free energy. This made the nucleation time increase less rapidly with the supercooling temperature and promoted nucleation. Because this latter effect is dominant, the net effect of the lysozyme is to promote nucleation.

ELIZABETH A. SHACK

\section{Possibility of the Formation of Mesostructured Silica Titania Mixed-Oxide Thin Films with High Titania Load Demonstrated}

The ability to prepare mesostructured thin films is a prerequisite to the implementation of many technological applications such as sensors, catalysts, and microelectronics. A group of researchers at the Institute of Materials Chemistry within the Vienna University of Technology, Austria, and the Advanced Materials Laboratory at the University of New Mexico has developed a method for the morphology-controlled synthesis of mesostructured silica titania mixed-oxide thin films with a high titanium load (Si:Ti ratio as high as 5:1) and good distribution of titanium atoms in the silica pore wall.

As reported in the June 17 issue of Chemistry of Materials, Nicola Hüsing of Vienna and co-workers used a titaniumcoordinated surfactant as a structuredirecting agent in their rapid and continuous technique for the formation of mesoporous silica titania mixed-metal-oxide thin films. The underlying principle is based on the evaporation-induced selfassembly of a modified amphiphile in combination with simultaneous sol-gel processing of the inorganic species. The researchers used the hydrophilic head group of oligo(ethylene oxide) alkyl ether surfactants $\left\{\right.$ Brij56 $\left[\mathrm{C}_{16} \mathrm{H}_{33}\left(\mathrm{OCH}_{2} \mathrm{CH}_{2}\right)_{10}\right.$ $\mathrm{OH}] /$ Brij92 $\left.\left[\mathrm{C}_{18} \mathrm{H}_{35}\left(\mathrm{OCH}_{2} \mathrm{CH}_{2}\right)_{2} \mathrm{OH}\right]\right\}$ for coordination to the titanium alkoxide. The thus-modified surfactant performs two essential functions, acting as both a structure-directing agent and a coordinating ligand to lower the reactivity of the titanium alkoxide.

Deposition of the thin films was performed by a dip-coating method from the sol. After calcination at $450^{\circ} \mathrm{C}$ for $3 \mathrm{~h}$, transparent, crack-free, and optically uniform silica titania films with a thickness of 200-250 nm were obtained. The researchers confirmed a well-defined, ordered structure and a good distribution of tetrahedrally coordinated titanium atoms in the silica pore walls by lowangle $\mathrm{x}$-ray diffraction, transmission electron microscopy, and UV- and Fourier transform infrared spectroscopy.

The researchers examined the thermal stability of the films in the temperature range of $450-1000^{\circ} \mathrm{C}$. After annealing at temperatures above $450^{\circ} \mathrm{C}$, amorphous titania converts to anatase and, later on (at $800^{\circ} \mathrm{C}$ ), to rutile. The research team concluded that the proposed approach opens the way to a variety of mesostructured mixed metal oxides.

ANDREI A. ELISEEV

\section{Spontaneous Emission from $\mathrm{CdSe} / \mathrm{CdS}$ Core-Shell Quantum Dots Modified in the Presence of a $\mathrm{SiO}_{2} / \mathrm{Si}$ Interface}

The ability to precisely control the optical and electronic properties of nanomaterials by simply changing their size has led to potential application of these materials in areas such as biological chips, solar-energy conversion, catalysis, and light-emitting diodes. The desire for greater understanding of the properties of these unique materials has spawned further research. A group of researchers led by Min Xiao in the Department of Physics at the University of Arkansas has demonstrated a method to control the photoluminescence (PL) intensity of CdSe nanocrystals by adjusting their position with respect to a $\mathrm{SiO}_{2} / \mathrm{Si}$ interface. They studied the spontaneous-emission lifetime of CdSe/CdS core-shell quantum dots as a function of distance between the dots and a polished Si surface. The researchers observed a damped oscillatory behavior in the lifetime of the PL as the separation distance between the nanomaterials and the surface was increased. According to Xiao, "Control of spontaneous emission from semiconductor structures may be useful in future applications of optoelectronic devices."

As described in the July 15 issue of Optics Letters, the researchers used CdSe nanocrystals, $4 \mathrm{~nm}$ in diameter and passivated with a CdS shell, to increase the PL efficiency of the materials. The nanomaterials were added to a solution of poly(methyl methacrylate) and spincoated onto a layer of $\mathrm{SiO}_{2}$ thermally grown on a silicon wafer. The thickness of the $\mathrm{SiO}_{2}$ layers was varied between $20 \mathrm{~nm}$ and $400 \mathrm{~nm}$. The researchers then excited the CdSe film with 400-nm laser radiation and measured the PL intensity decay at $605 \mathrm{~nm}$ through the use of a time-correlated photon counting system.

The researchers found that the spontaneous-emission lifetime showed a damped oscillatory dependence on the separation distance between the nano- 